\title{
Esmolol for cardioprotection during resuscitation with adrenaline in an ischaemic porcine cardiac arrest model
}

Hilde Karlsen ${ }^{1,2^{*}}$, Harald Arne Bergan ${ }^{3}$, Per Steinar Halvorsen ${ }^{2,4}$, Kjetil Sunde ${ }^{3,4}$, Eirik Qvigstad ${ }^{5}$, Geir Øystein Andersen ${ }^{5}$, Jan Frederik Bugge ${ }^{3^{\wedge}}$ and Theresa Mariero Olasveengen ${ }^{3,6}$

* Correspondence: hildekar@gmail.
com
'Department of Research and
Development, Oslo University
Hospital, PB 4956 Nydalen, N-0424
Oslo, Norway
2The Intervention Center,
Department of Anaesthesiology,
Oslo University Hospital, Oslo,
Norway
Full list of author information is
available at the end of the article

\begin{abstract}
Background: The effectiveness of adrenaline during resuscitation continues to be debated despite being recommended in international guidelines. There is evidence that the $\beta$-adrenergic receptor (AR) effects of adrenaline are harmful due to increased myocardial oxygen consumption, post-defibrillation ventricular arrhythmias and increased severity of post-arrest myocardial dysfunction. Esmolol may counteract these unfavourable $\beta$-AR effects and thus preserve post-arrest myocardial function. We evaluated whether a single dose of esmolol administered prior to adrenaline preserves post-arrest cardiac output among successfully resuscitated animals in a novel, ischaemic cardiac arrest porcine model.
\end{abstract}

Methods: Myocardial infarction was induced in 20 anaesthetized pigs by inflating a percutaneous coronary intervention (PCl) balloon in the circumflex artery 15 min prior to induction of ventricular fibrillation. After 10 min of untreated VF, resuscitation with veno-arterial extracorporeal membrane oxygenation (VA-ECMO) was initiated and the animals were randomized to receive an injection of either $1 \mathrm{mg} / \mathrm{kg}$ esmolol or $9 \mathrm{mg} / \mathrm{ml} \mathrm{NaCl}$, prior to adrenaline. Investigators were blinded to allocation.

Successful defibrillation was followed by a 1-h high-flow VA-ECMO before weaning and an additional 1-h stabilization period. The PCI-balloon was deflated 40 min after inflation. Cardiac function pre- and post-arrest (including cardiac output) was assessed by magnetic resonance imaging (MRI) and invasive pressure measurements. Myocardial injury was estimated with MRI, triphenyl tetrazolium chloride (TTC) staining and serum concentrations of cardiac troponin T.

Results: Only seven esmolol and five placebo-treated pigs were successfully resuscitated and available for post-arrest measurements $(p=0.7)$. MRI revealed severe but similar reductions in post-arrest cardiac function with cardiac output $3.5(3.3,3.7)$ and $3.3(3.2,3.9) \mathrm{l} / \mathrm{min}$ for esmolol and control (placebo) groups, respectively $(p=0.7)$. The control group had larger left ventricular end-systolic and end-diastolic ventricular volumes compared to the esmolol group $(75(65,100)$ vs. $62(53,70) \mathrm{ml}, p=0.03$ and $103(86,124)$ vs. $87(72,91) \mathrm{ml}, p=0.03$ for control and esmolol groups, respectively). There were no other significant differences in MRI characteristics, myocardial infarct size or other haemodynamic measurements between the two groups.

(Continued on next page) 
(Continued from previous page)

Conclusions: We observed similar post-arrest cardiac output with and without a single dose of esmolol prior to adrenaline administration during low-flow VA-ECMO in an ischaemic cardiac arrest pig model.

Keywords: Resuscitation, Cardiopulmonary resuscitation, Esmolol, $\beta$-adrenergic blocker, Extra-corporal membrane oxygenation (ECMO), Porcine model, Myocardial infarction, Cardiac MRI, Cardioprotection

\section{Background}

Cardiac arrest remains a major public health problem with an estimated 100 per 100, 000 Europeans affected every year. Although cardiac arrest management and training are constantly evolving, only on average $10 \%$ of patients who suffer out-of-hospital cardiac arrest (OHCA) can currently be expected to survive the event [1]. Current initial treatment for cardiac arrest involves high-quality chest compressions, ventilations, and timely defibrillations for those with shockable rhythms [2]. Drugs like adrenaline, amiodarone and to some extent lidocaine are also recommended in current European treatment guidelines [3], but their efficiency remains heavily debated. There is an urgent need for more effective drugs to improve both initial resuscitation and longterm outcomes after cardiac arrest.

Adrenaline has potent vasoconstrictive $\alpha$-adrenergic receptor (AR) effects that have been consistently shown to increase coronary perfusion pressure (CPP) and return of spontaneous circulation (ROSC) in laboratory studies [4-6]. Randomized controlled trials on adrenaline have confirmed the drug's ability to improve ROSC rates, but have largely failed to translate the large improvements in ROSC into long-term survival with good neurological outcome [7-9]. It is questioned whether the coveted $\alpha$-AR effects are negated by unwanted $\beta$-AR effects, such as increased myocardial oxygen consumption [10], post-arrest ventricular arrhythmias $[11,12]$ and post-arrest myocardial dysfunction $[13,14]$. Counteracting $\beta-A R$ stimulation with a $\beta-A R$ antagonist has been shown to be cardioprotective, anti-arrhythmic and increase ROSC in experimental models where adrenaline has been administered during cardiopulmonary resuscitation (CPR) [15-21].

Esmolol is a selective ultrashort-acting $\beta_{1}-\mathrm{AR}$ blocking agent producing competitive blockade of $\beta_{1}-$ ARs in both animals and humans[22]. Its short half-life of $9.2 \mathrm{~min}$ makes it uniquely suited as a resuscitation drug where heart rhythms and haemodynamic are unstable and shift rapidly. In a recent case series, esmolol given to patients with refractory ventricular fibrillation (VF) was reported to result in higher ROSC rates and improved survival compared to those who did not receive esmolol [23]. All previous experimental studies on $\beta$-AR blockers in cardiac arrest have been performed in arrhythmia models [15-21], while the potential effect of antagonizing the detrimental effects of $\beta$-AR stimulation by adrenaline is probably most pronounced in ischaemic hearts. Thus, the aim of this randomized, placebo-controlled study was to evaluate the effect of esmolol administered before adrenaline in an ischaemic porcine cardiac arrest model. We used a single dose of esmolol in order to explore a study design that could be easily adapted to a clinical OHCA setting. We hypothesized that a single dose of esmolol administered before adrenaline would increase post-arrest cardiac function. 
Our primary outcome was post-arrest cardiac output, whereas secondary outcomes included initial resuscitation success, myocardial injury and additional post-arrest cardiac function parameters.

\section{Methods}

Design

This was a blinded block-randomized placebo-controlled intervention study in pigs to compare initial resuscitation success and post-arrest cardiac function with intravenous esmolol (Brevibloc ${ }^{\circ} 10 \mathrm{mg} / \mathrm{ml}$, Baxter, Deerfield, IL, USA) versus control $(9 \mathrm{mg} / \mathrm{ml} \mathrm{NaCl})$ during resuscitation with veno-arterial extracorporeal membrane oxygenation (VAECMO) following acute myocardial infarction and cardiac arrest. Non-participating personnel had access to the randomization list and prepared the study drug. Investigators remained blinded to randomization until all data were collected and analysed. The study was performed at The Intervention Center, Oslo University Hospital. Equipment used in the experiments is presented in detail in the additional materials.

\section{Anaesthesia and animal preparation}

We included 20 healthy crossbreed Norwegian Landrace pigs (48 $\mathrm{kg}$ (range 46-51)) of either sex in the study. The animals were anaesthetized and surgically prepared as previously described in detail [24]. In brief, after fasting overnight aside from free water access, the animals were pre-medicated in the animal facility (Department of Comparative Medicine, University of Oslo) by an intramuscular injection with a mixture of $30 \mathrm{ml}$ ketamine 50 $\mathrm{mg} / \mathrm{ml}(30 \mathrm{mg} / \mathrm{kg}), 4 \mathrm{ml}$ azaperone $40 \mathrm{mg} / \mathrm{ml}(3 \mathrm{mg} / \mathrm{kg})$ and $1 \mathrm{ml}$ atropine $1 \mathrm{mg} / \mathrm{ml}(20 \mu \mathrm{g} /$ $\mathrm{kg}$ ) and then transported to the operating theatre. Further anaesthesia was provided with a weight standardized mixture of pentobarbital $4 \mathrm{mg} / \mathrm{kg} / \mathrm{h}$, morphine $2 \mathrm{mg} / \mathrm{kg} / \mathrm{h}$ and midazolam $0.15 / \mathrm{kg} / \mathrm{h}$ suspended in Ringer`s acetate solution infused at $10 \mathrm{ml} / \mathrm{kg} / \mathrm{h}$. Rocuronium was infused at $3 \mathrm{mg} / \mathrm{kg} / \mathrm{h}$. The pigs were mechanically ventilated with tidal volume $10 \mathrm{ml} /$ $\mathrm{kg}$, respiratory rate (RR) 18/min, positive end-expiratory pressure (PEEP) $5 \mathrm{mmH}_{2} \mathrm{O}$, fraction of inspired oxygen 0.4 and ratio of inspiratory to expiratory time (I:E ratio) $1: 2 . \mathrm{PaO}_{2}$ and $\mathrm{PaCO}_{2}$ were adjusted according to blood gas analyses throughout the experiment.

The surgical preparation included a tracheostomy and placement of a left intraventricular pressure catheter, a carotid arterial pressure catheter and a pulmonary artery (PA) catheter. In addition, ECMO cannulas were positioned through the right internal jugular vein and the left femoral artery [24].

\section{Experimental protocol (Fig. 1)}

After the surgical preparation and a following 30-min stabilization period, pre-arrest (baseline) haemodynamic measurements and cardiac MRI were obtained and blood samples were withdrawn for analyses of serum concentrations of cardiac Troponin $\mathrm{T}$ (cTnT) and aspartate transaminase (ASAT).

The pigs were connected to the Ringer's acetate primed VA-ECMO circuit with the circuitry set to stand-by and the vascular connections clamped. An intravenous injection of heparin $2 \mathrm{mg} / \mathrm{kg}$ followed by $0.5 \mathrm{mg} / \mathrm{kg} / \mathrm{h}$ infusion was provided to prevent blood clotting in the ECMO circuit. Activated clotting time (ACT) was targeted to $\geq 300 \mathrm{~s}$. 


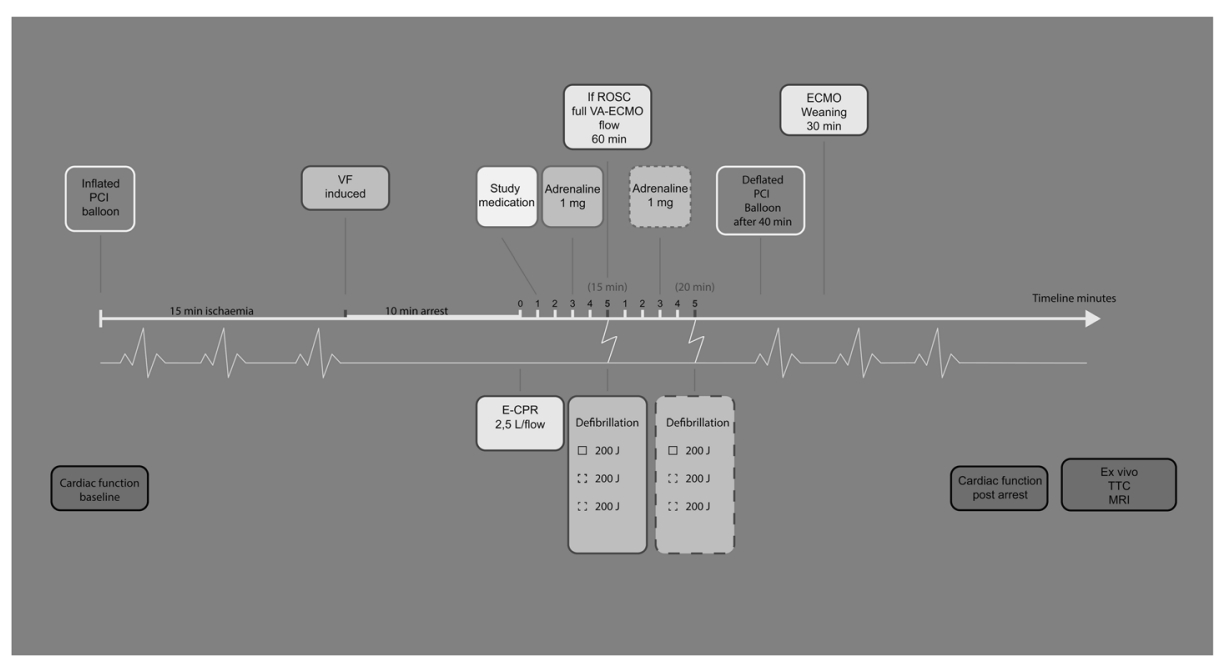

Fig. 1 Experimental timeline. Overview of the experiment. PCl balloon percutaneous coronary intervention balloon, VF ventricular fibrillation, E-CPR extracorporeal cardiopulmonary resuscitation, ROSC return of spontaneous circulation, ECMO extracorporeal membrane oxygenation

A myocardial infarction was induced by fluoroscopy-guided intravascular balloon occlusion of the proximal coronary circumflex artery. After $15 \mathrm{~min}$ of occlusion, VF was induced using a $9 \mathrm{~V}$ trans-thoracic current device and confirmed by electrocardiography (ECG) and a rapid aortic arterial pressure drop. The VF was left untreated for 10 min with ventilation on hold. Resuscitation with low-flow $(2.5 \mathrm{l} / \mathrm{min})$ VA-ECMO was then initiated and mechanical ventilation restarted with RR set to $10 / \mathrm{min}$. VA-ECMO flow rate was selected to mimic flow generated by optimal CPR. Identical volumes of study drug, (either esmolol $1 \mathrm{mg} / \mathrm{kg}$ or $\mathrm{NaCl} 9 \mathrm{mg} / \mathrm{ml}$-control) were administered 1 min after the initiation of VA-ECMO, 2 min prior to $1 \mathrm{mg}$ adrenaline administration and $4 \mathrm{~min}$ prior to the first defibrillation attempt to allow adequate circulation of drugs. The first attempt to terminate VF was thereby initiated $15 \mathrm{~min}$ after VF induction using biphasic 200-Joule defibrillation(s) (maximum 3). If the first defibrillation failed, adrenaline was re-administered after $3 \mathrm{~min}$, and defibrillation(s) (maximum three) was repeated $2 \mathrm{~min}$ after adrenaline injection. The study protocol allowed a maximum of two 5-min cycles with adrenaline and up to six defibrillations. Pilot experiments suggested resuscitation beyond $10 \mathrm{~min}$ of ECMO and six defibrillations to be futile. The balloon in the circumflex artery was deflated after a 40-min occlusion-time.

Successful defibrillation was followed by a 1-h ECMO-support at the maximum achievable ECMO blood flow. During ECMO-support, lower limits of mean arterial pressure (MAP) and pulse pressure were $50 \mathrm{mmHg}$ and $15 \mathrm{mmHg}$, respectively, sustained if necessary by fluid ( $\max 11$ of Ringer's acetate) and dobutamine infusions (maximum $5 \mu \mathrm{g} / \mathrm{kg} /$ $\mathrm{min}$ ). The ECMO-support was reduced by $1 / 6$ of maximum flow every 5 min during a 30 min weaning period in accordance with a standardized weaning protocol. The total VAECMO duration was median $100 \mathrm{~min}$ for the esmolol group (range 95-110 min) and median 95 min for the placebo group (range 95-103 min). After successful ECMO weaning, dobutamine was discontinued. Post-arrest haemodynamic measurements and cardiac MRI were re-assessed after an additional 30-min stabilization period. Analysis of serum concentrations of $\mathrm{cTnT}$ and ASAT were also repeated post-arrest. 
The animals were euthanized with an intravenous injection of potassium chloride 1 $\mathrm{mmol} / \mathrm{kg}$, morphine $1 \mathrm{mg} / \mathrm{kg}$ and pentobarbital $20 \mathrm{mg} / \mathrm{kg}$. Immediately post-mortem, a median sternotomy was performed and the heart was excised, sliced and stained in triphenyl tetrazolium chloride (TTC) to estimate myocardial infarction size.

\section{Cardiac function assessments}

Arterial, pulmonary artery and left ventricular pressures were measured continuously. Maximum and minimum left ventricular pressure pressures $\left(\mathrm{LVP}_{\max }, \mathrm{LVP}_{\min }\right)$ and the related first time-derivate of LVP ( $\mathrm{LV} \mathrm{dP} / \mathrm{dt}_{\max }$ and $\left.\mathrm{dP} / \mathrm{dt}_{\min }\right)$ were also determined. Left ventricle (LV) function at baseline and post-arrest were assessed by MRI and included measurements of stroke volume (SV), cardiac output ( $\mathrm{CO}=\mathrm{SV} \times$ heart rate $(\mathrm{HR}))$, end-diastolic volume (EDV), end-systolic volume (ESV), ejection fraction (EF = SV/EDV), mitral annular plane excursion (MAPSE) and mid-LV radial wall thickening.

Cardiac MRI was performed as previously described in detail [24], and in accordance with the recommendations from the Society for Cardiovascular Magnetic Resonance [25]. Cardiac output was assessed by phase-contrast imaging with blood velocity encoded through-plane images of the mid-ascending aorta, a technique considered to provide robust and accurate cardiac output measurements [24, 26]. The images were analysed using the software Medviso Segment version 2.1 R6005 (http://segment.heiberg.se) [27]. Intra- and inter-observer analyses were performed.

\section{MRI-assessed infarct size and histological staining}

Before euthanasia, each pig received a dose of gadolinium contrast medium to enhance the infarcted area on the ex vivo MRI scans of the heart. A combination of manually drawing and auto-detection tool was used to estimate myocardial infarct size with the software Medviso Segment version 2.1 R6005 [27].

After euthanasia and post-mortem MRI, the left ventricle was excised and cut into approximately $0.5 \mathrm{~cm}$ thick slices before staining in tetrazolium chloride (TTC) at $38^{\circ} \mathrm{C}$ for $20 \mathrm{~min}$. Infarct size was determined as percentage of the left ventricle [28, 29] using Photoshop CC2017, version 18.01.

\section{Statistics}

Statistical analyses were performed using IBM SPSS Statistics Software version 25. Values are reported as medians with $95 \%$ confidence intervals. Groups were compared using Pearson's chi-squared test or Wilcoxon-Mann-Whitney U test as appropriate. Based on initial pilot and previous experiments, we estimated that post-arrest cardiac output would be $3.3 \pm 0.5 \mathrm{l} / \mathrm{min}$ in the control group. Assuming cardiac output improved by $15-20 \%$ with esmolol (up to $4.0 \pm 0.5 \mathrm{l} / \mathrm{min}$ ), we estimated needing approximately 10 animals in each group with $\alpha=0.05$ and power $1-\beta=0.9$.

\section{Interobserver and intraobserver variability}

Four different MRI baseline measurements and two different MRI post-arrest measurements were randomly selected to investigate inter- and intraobserver variability of ejection fraction and cardiac output. The intraclass correlation coefficient of inter- and intraobserver variability for ejection fraction were 0.96 (95\% CI $0.7-1.0 ; p<0.001)$ and 
0.98 (95\% CI $0.87-1.0 ; p<0.001$ ), respectively, and for cardiac output 1.0 and 1.0 (95\% CI 0.99-1.0), respectively.

\section{Results}

There were no differences in baseline characteristics between the two groups. The esmolol group consisted of six males and four females with average weight $48 \mathrm{~kg}$ (95\% CI 47,51 ), and placebo group seven males and three females with average weight $48 \mathrm{~kg}$ (95\% CI 46, 51). Baseline haemodynamic, blood gas values and serum levels of cTNT and ASAT are shown in Table 1.

\section{Post-arrest cardiac function}

All animals had significantly reduced cardiac function post-arrest, but there were no significant differences in our primary outcome, cardiac output, between the esmolol and placebo groups $(3.5(3.3,3.7)$ vs. $3.3(95 \%$ CI $3.2,3.9)$, respectively, $p=0.7)$. There were no differences in arterial, central venous, pulmonary artery or left ventricular pressures between the two groups post-arrest. In addition, there was no difference in HR or in contraction, expressed as $\mathrm{dp} / \mathrm{dt}_{\max }$, and relaxation, expressed as $\mathrm{dp} / \mathrm{dt}_{\min }$ (Fig. 2, Table 1). Both left ventricular end-systolic and end-diastolic volumes were significantly lower in the esmolol vs the control group; median $62(95 \%$ CI 53, 70) vs. $75(95 \%$ CI 65, 100) ml, $p=$ 0.03 and 87 (95\% CI 72, 91) vs. 103 (95\% CI 86, 124) ml, $p=0.04$, respectively). Despite differences in ventricular volumes between the two groups, there were no significant differences in any of the other cardiac function parameters. (Fig. 3, Table 1)

\section{Initial resuscitation}

All animals in the esmolol group and 9 of 10 animals in the control group had ROSC $(p=0.3)$, but only 7 of 10 esmolol animals and 5 of 10 control animals were successfully weaned from ECMO ( $p=0.7)$. The two groups received similar numbers of defibrillations; median 3 (95\% CI 1, 7) vs. 4 (95\% CI 1, 9) for esmolol and control groups, respectively, $(p=0.9)$, received the same amount of adrenaline ( $2 \mathrm{mg}$ for both esmolol and control groups, respectively) and 7 of 10 animals in both groups needed transient post-arrest dobutamine infusions. In addition, 2 of 5 in the control group and 5 of 7 in the esmolol group needed dobutamine infusion during the weaning and stabilization phase, discontinued before post-arrest measurements. Only one animal, in the esmolol group, needed dobutamine infusion throughout the experiment (Table 2).

\section{Post-arrest injury}

Infarct size was similar in the two groups as evaluated with both TTC staining and MRI. With TTC staining infarct size was median 20 (95\% CI 16, 30) vs. 25 (95\% CI 9, 34 ) \% of the left ventricle for esmolol and control groups, respectively, $\mathrm{p}=1.0$, and with MRI median $20(95 \%$ CI 18, 32) vs. $22(95 \%$ CI 7, 25) \% of left ventricle for esmolol and control groups, respectively, $p=0.4$ (Table 1). There were no differences in markers of myocardial injury (ASAT and cTNT) between the two groups (Table 1). Post-arrest $\mathrm{SvO}_{2}$ values were low for both groups, but significantly lower in the esmolol group; median $33(95 \%$ CI 23, 41) vs. 38 (95\% CI 35, 45) \% for control group, $p=0.04)$ (Table 1). Lactate values were within the upper normal range for both groups; median 
Table 1 Haemodynamic-, MRI-, and blood-gas measurements. Values are expressed as medians with 95\% confidence intervals (lower limit, higher limit). Groups are compared using WilcoxonMann-Whitney $U$ test. MRI magnetic resonance imaging, HR heart rate, MAP mean arterial pressure, MPAP mean pulmonary artery pressure, Wedge pulmonary wedge pressure, CVP central venous pressure, $L V P_{\text {max }}$ maximum left ventricle (LV) pressure, $d P / d t_{\max }$ maximum $L V$ pressure first time derivate, $d P / d t_{\min }$ minimum LV pressure first time derivate, $L V$ ESP left ventricular end-systolic pressure, LV EDP left ventricular end-diastolic pressure, COphase cardiac output by cardiac MRI phase-contrast technique, LV EDV left ventricular end-diastolic volume, LV ESV left ventricular endsystolic volume, Wall thickening septum and Wall thickening lat mid-left ventricular radial wall thickening in septum and lateral wall, MAPSE mitral annular plane systolic excursion , LV EF left ventricular ejection fraction. $\mathrm{SVO}_{2}$ mixed venous oxygen saturation, ASAT aspartate transaminase, CTnT cardiac troponin T, TTC triphenyl tetrazolium chloride tissue-staining, LV left ventricle

\begin{tabular}{|c|c|c|c|c|c|}
\hline \multirow[t]{2}{*}{ Variable } & \multicolumn{2}{|l|}{ Esmolol-group } & \multicolumn{2}{|l|}{ Control group } & \multirow{2}{*}{$\begin{array}{l}P \text { value } \\
\text { Difference } \\
\text { between } \\
\text { groups } \\
\text { post-arrest }\end{array}$} \\
\hline & $\begin{array}{l}\text { Baseline } \\
(n=10)\end{array}$ & $\begin{array}{l}\text { Post-arrest } \\
(n=7)\end{array}$ & $\begin{array}{l}\text { Baseline } \\
(n=10)\end{array}$ & $\begin{array}{l}\text { Post-arrest } \\
(n=5)\end{array}$ & \\
\hline \multicolumn{6}{|l|}{ Haemodynamic variables } \\
\hline HR (beats/min) & $78(69,89)$ & $137(95,180)$ & $70(66,88)$ & $114(90,142)$ & 0.3 \\
\hline MAP (mmHg) & $96(78,112)$ & $75(62,88)$ & $92(80,103)$ & $79(60,84)$ & 0.9 \\
\hline MPAP (mmHg) & $22(18,25)$ & $21(15,28$ & $21(16,25)$ & $24(17,30)$ & 0.6 \\
\hline Wedge (mmHg) & $14(11,17)$ & $13(11,23)$ & $13(10,24)$ & $20(4,23)$ & 0.3 \\
\hline CVP $(\mathrm{mmHg})$ & $8(6,12)$ & $7(4,11)$ & $9(8,12)$ & $9(7,12)$ & 0.4 \\
\hline LVPmax (mmHg) & $106(93,120)$ & $89(72,105)$ & $110(95,117)$ & $90(71,98)$ & 1.0 \\
\hline LV ESP (mmHg) & $62(53,80)$ & $52(41,58)$ & $65(53,75)$ & $57(41,62)$ & 0.2 \\
\hline LV EDP (mmHg) & $16(11,19)$ & $16(6,24)$ & $16(14,21)$ & $19(15,28)$ & 0.2 \\
\hline $\mathrm{LV} \mathrm{dp} / \mathrm{dt}_{\max }(\mathrm{mmHg} / \mathrm{s})$ & $\begin{array}{l}1771 \\
(1248,2448)\end{array}$ & $\begin{array}{l}1916 \\
(1322,3691)\end{array}$ & $\begin{array}{l}1677 \\
(1349,1989)\end{array}$ & $\begin{array}{l}1486 \\
(845,1870)\end{array}$ & 0.1 \\
\hline $\mathrm{LV} \mathrm{dp} / \mathrm{dt}_{\min }(\mathrm{mmHg} / \mathrm{s})$ & $\begin{array}{l}-2339 \\
(-1756,-2664)\end{array}$ & $\begin{array}{l}-2108 \\
(-1163,-2944)\end{array}$ & $\begin{array}{l}-2479 \\
(-1608,-3624)\end{array}$ & $\begin{array}{l}-1586 \\
(-1375,-2261)\end{array}$ & 0.3 \\
\hline \multicolumn{6}{|l|}{ MRI measurements } \\
\hline COphase (I/min) & $4.8(4.2,5.6)$ & $3.5(3.3,3.7)$ & $4.9(4.6,5.3)$ & $3.3(3.2,3.9)$ & 0.7 \\
\hline LV SV (ml) & $61(55,69)$ & $27(18,39)$ & $69(62,75)$ & $31(20,36)$ & 0.4 \\
\hline LV EDV (ml) & $108(104,132)$ & $87(72,91)$ & $117(108,130)$ & $103(86,124)$ & 0.04 \\
\hline LV ESV (ml) & $53(46,69)$ & $62(53,70)$ & $50(42,62)$ & $75(65,100)$ & 0.03 \\
\hline LV EF (\%) & $50(41,66)$ & $26(21,33)$ & $60(44,65)$ & $25(19,29)$ & 0.5 \\
\hline $\begin{array}{l}\text { Wall thickening septum } \\
\text { (\%) }\end{array}$ & $35(27,42)$ & $21(1,37)$ & $35(26,53)$ & $32(1,45)$ & 1.0 \\
\hline $\begin{array}{l}\text { Wall thickening lateral } \\
\text { (\%) }\end{array}$ & $35(26,48)$ & $26(12,32)$ & $42(23,48)$ & $9(9,47)$ & 0.4 \\
\hline MAPSE (mm) & $14.6(13.4,14.6)$ & $6.3(5.4,7.5)$ & $13.4(13.7,14.3)$ & $6.6(5.8,8.6)$ & 1.0 \\
\hline \multicolumn{6}{|l|}{ Blood gas analysis } \\
\hline $\mathrm{Hb}(\mathrm{g} / \mathrm{dl})$ & $8.4(7.6,9.8)$ & $9.0(7.5,15.2)$ & $8.8(7.8,9.3)$ & $9.3(7.4,10.0)$ & 1.0 \\
\hline $\mathrm{PaO}_{2}(\mathrm{kPa})$ & $23.4(20.9,28.2)$ & $23.9(20.1,34.4$ & $24.9(20.5,26.2)$ & $25.3(23.9,26.9)$ & 0.9 \\
\hline $\mathrm{PaCO}_{2}(\mathrm{kPa})$ & $4.7(4.4,5.4)$ & $4.6(2.5,5.2)$ & $4.8(4.4,4.9)$ & $4.5(4.4,4.8)$ & 0.9 \\
\hline $\mathrm{pH}$ & $7.55(7.52,7.60)$ & $7.50(7.50,7.80)$ & $7.55(7.50,7.60)$ & $7.50(7.50,7.50)$ & 0.4 \\
\hline Lactate (mmol/l) & $1.0(0.6,1.5)$ & $2.4(0.7,3.7$ & $0.7(0.7,1.0)$ & $2.3(2.1,2.9)$ & 0.9 \\
\hline $\mathrm{SvO}_{2}(\%)$ & $58(37,76)$ & $33(23,41)$ & $57(55,65)$ & $38(35,45)$ & 0.04 \\
\hline \multicolumn{6}{|l|}{ Myocardial injury } \\
\hline ASAT (U/I) & $31(21,46)$ & $309(190,542)$ & $24(21,28)$ & $354(136,1567)$ & 0.11 \\
\hline cTnT (ng/l) & $11(6,46)$ & $\begin{array}{l}3864 \\
(2052,15727)\end{array}$ & $15(13,19)$ & $\begin{array}{l}4014 \\
(1018,8420)\end{array}$ & 0.5 \\
\hline
\end{tabular}


Table 1 Haemodynamic-, MRI-, and blood-gas measurements. Values are expressed as medians with 95\% confidence intervals (lower limit, higher limit). Groups are compared using WilcoxonMann-Whitney $U$ test. $M R I$ magnetic resonance imaging, HR heart rate, MAP mean arterial pressure, MPAP mean pulmonary artery pressure, Wedge pulmonary wedge pressure, CVP central venous pressure, $L V P_{\text {max }}$ maximum left ventricle (LV) pressure, $d P / d t_{\max }$ maximum $L V$ pressure first time derivate, $d P / d t_{\min }$ minimum LV pressure first time derivate, LV ESP left ventricular end-systolic pressure, LV EDP left ventricular end-diastolic pressure, COphase cardiac output by cardiac MRI phase-contrast technique, LV EDV left ventricular end-diastolic volume, LV ESV left ventricular endsystolic volume, Wall thickening septum and Wall thickening lat mid-left ventricular radial wall thickening in septum and lateral wall, MAPSE mitral annular plane systolic excursion , LV EF left ventricular ejection fraction. $\mathrm{SVO}_{2}$ mixed venous oxygen saturation, ASAT aspartate transaminase, CTnT cardiac troponin T, TTC triphenyl tetrazolium chloride tissue-staining, $L V$ left ventricle (Continued)

\begin{tabular}{|c|c|c|c|c|c|}
\hline \multirow[t]{2}{*}{ Variable } & \multicolumn{2}{|c|}{ Esmolol-group } & \multicolumn{2}{|c|}{ Control group } & \multirow{2}{*}{$\begin{array}{l}P \text { value } \\
\text { Difference } \\
\text { between } \\
\text { groups } \\
\text { post-arres }\end{array}$} \\
\hline & $\begin{array}{l}\text { Baseline } \\
(n=10)\end{array}$ & $\begin{array}{l}\text { Post-arrest } \\
(n=7)\end{array}$ & $\begin{array}{l}\text { Baseline } \\
(n=10)\end{array}$ & $\begin{array}{l}\text { Post-arrest } \\
(n=5)\end{array}$ & \\
\hline $\begin{array}{l}\text { Infarct size by MRI } \\
\text { (\% of LV) }\end{array}$ & $\ldots$ & $20(18,32)$ & $\ldots$ & $22(7,25)$ & 0.4 \\
\hline $\begin{array}{l}\text { Infarct size by TTC } \\
\text { (\% of LV) }\end{array}$ & $\ldots$ & $20(16,30)$ & $\ldots$ & $25(9,34)$ & 1.0 \\
\hline
\end{tabular}

$2.4(95 \%$ CI $0.7,3.7)$ vs. 2.3 (95\% CI $2.1,2.9) \mathrm{mmol} / \mathrm{l}$ for esmolol and control groups, respectively, $p=0.9$ (Table 2 ).

\section{Discussion}

This study used an acute ischaemic porcine cardiac arrest model to investigate whether a single dose of esmolol given during the initial phase of resuscitation, and prior to administration of adrenaline, could improve initial resuscitation success and post-arrest cardiac function. Although $70 \%$ of the animals were successfully resuscitated in the esmolol group compared to $50 \%$ in the control group, the study was aimed and powered to evaluate post-arrest cardiac output, not survival outcomes, and therefore unable to demonstrate a statistical difference on resuscitation success. While we observed statistically significant differences in end-systolic and diastolic volumes and mixed central venous oxygen saturation, we were unable to demonstrate that esmolol protects against post-arrest cardiac dysfunction defined as post-arrest cardiac output. As very many parameters were compared between the two groups, there is also an increasing risk that some of these parameters will be statistically different by pure chance, and that the statistically significant differences observed may simply be related to the multiple comparisons between the two groups. Due to the exploratory design of the study, statistical correction for multiple comparisons was not performed.

Pre-arrest $\beta$-AR-blocker use has been associated with improved survival in patients with VF [30], and patients with implanted cardioverter defibrillators (ICDs) on previous $\beta$-AR blocker medication have been observed to experience fewer ICD defibrillations compared to those not using $\beta$-AR -blockers [31]. Additionally, two recent case series both reported better outcomes for a group of refractory VF cardiac arrest patients which received esmolol compared to a group that did not [23, 32]. Although the authors attempted to match patients who received esmolol to similar patients that did not, there is obvious risk of bias and unrecognized confounding in such non- 


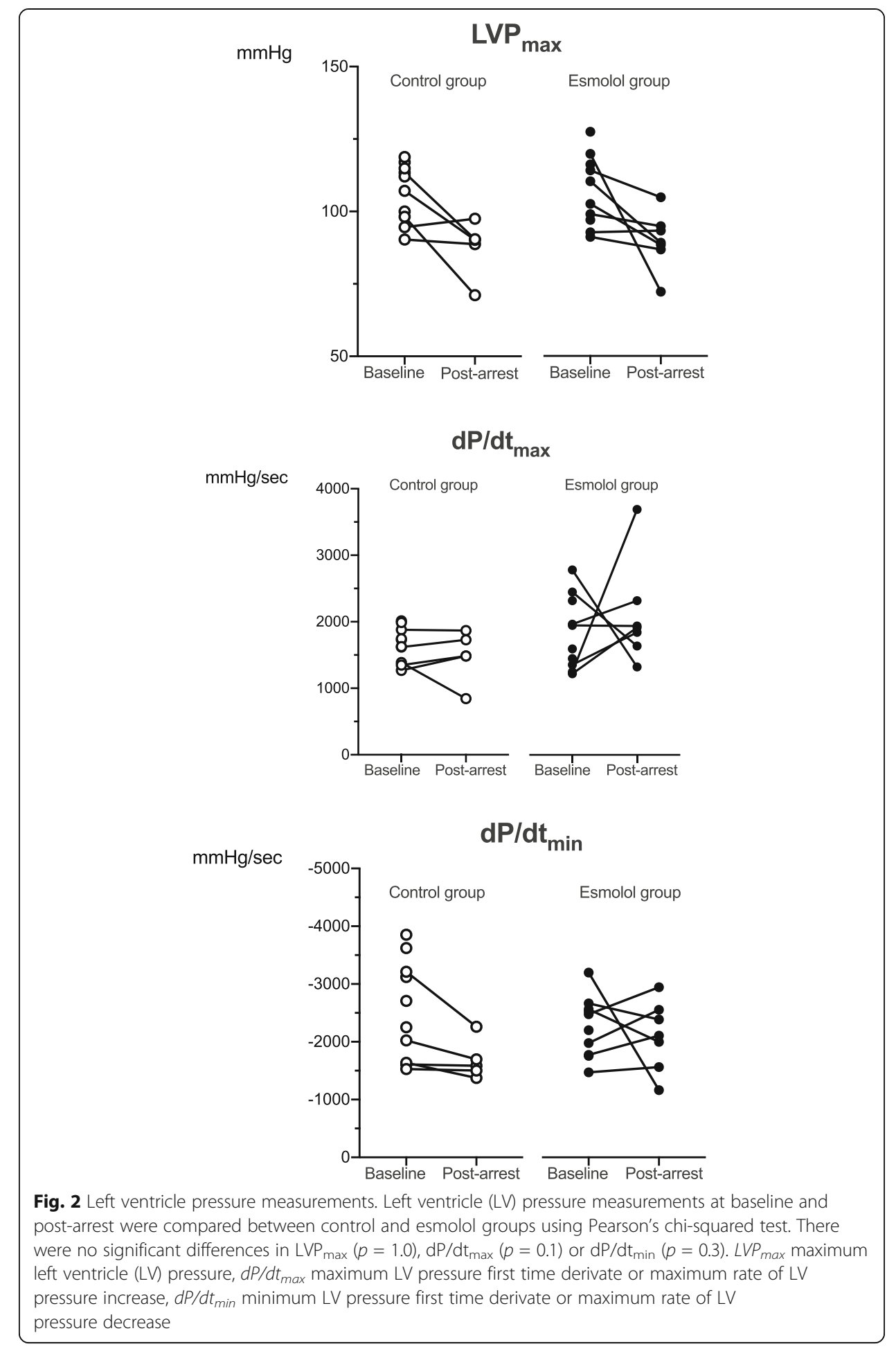

randomized designs. Nonetheless, these observations are interesting and warrant further exploration.

Although generally encouraging, previous experimental studies on esmolol during cardiac arrest have been divergent. Some studies have observed improved ROSC rates with fewer defibrillations in animals given esmolol in addition to adrenaline compared 


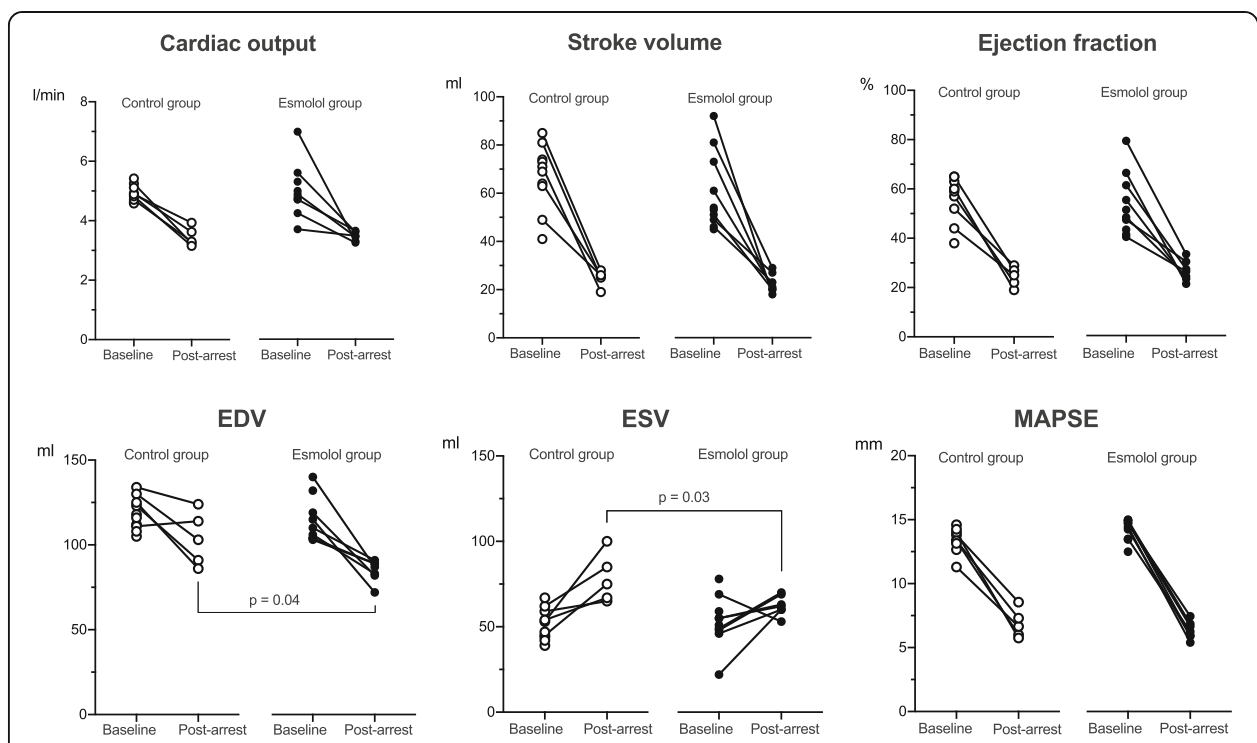

Fig. 3 Cardiac MRI measurements. Cardiac magnetic resonance imaging (MRI) measurements of the left ventricle at baseline and post-arrest were compared between control and esmolol groups. Data were compared using the Wilcoxon-Mann-Whitney U test. EDV left end-diastolic volume, ESV left end-systolic volume, MAPSE mitral annular plane systolic excursion

to animals given adrenaline alone $[14,15,18,20,33]$, whereas other studies have been unable to demonstrate a clear benefit from esmolol during initial resuscitation [34-37]. Similarly, some studies have reported impressive improvements in post-arrest cardiac function with esmolol $[14,16,18]$, while other studies have failed to observe any differences in relevant haemodynamic variables [34-37]. These conflicting results reflect the complexities of designing clinically relevant and robust experimental models, as well as raise the question whether (a) esmolol might not be a useful resuscitation drug or (b) we have not elucidated optimal timing, dose or form of administration.

If esmolol is beneficial during resuscitation from cardiac arrest, continuous infusion strategies where esmolol may be titrated to specific individual targets, (e.g. heart rate) could potentially be more effective than simpler bolus strategies. However, such strategies would effectively exclude use in ordinary prehospital settings where most cardiac arrests occur. As such, an effective bolus strategy has the greatest potential to translate from the experimental laboratory setting into clinical practice. The studies previously

Table 2 Outcome of resuscitation and pharmacological support. Values are expressed as medians with 95\% confidence intervals (lower limit, higher limit). Groups are compared using WilcoxonMann-Whitney test $U$ test for continuous variables and Pearson's chi-squared test for categorical variables. ROSC Return of spontaneous circulation

\begin{tabular}{llll}
\hline Variable & $\begin{array}{l}\text { Esmolol-group } \\
(n=10)\end{array}$ & $\begin{array}{l}\text { Control group } \\
(n=10)\end{array}$ & $\begin{array}{c}p \\
\text { value }\end{array}$ \\
\hline Number of defibrillations & $3(1,7)$ & $4(1,9)$ & 0.9 \\
Successful defibrillation & 10 of 10 & 9 of 10 & 0.3 \\
Sustained ROSC & 7 of 10 & 5 of 10 & 0.7 \\
Adrenaline dose $(\mathrm{mg})$ & $2(1,2)$ & $2(1,2)$ & 1.0 \\
Need for dobutamine post-arrest & 5 of 7 & 2 of 5 & 0.3 \\
Dobutamine dose $(\mathrm{mg})$ & $4.7(3,59)$ & $8.5(3,14)$ & 1.0 \\
\hline
\end{tabular}


demonstrating benefits from esmolol during resuscitation in experimental animal models have used single doses of $0.3-1 \mathrm{mg} / \mathrm{kg}[14-16,18,20,33]$ administered at the beginning of the resuscitation effort, comparable to the present $1 \mathrm{mg} / \mathrm{kg}$ dose. However, a previous dose-exploring study evaluating cardioprotective effects of esmolol during cardiac surgery suggested that $0.25 \mathrm{mg} / \mathrm{kg}$ was superior to $0.5 \mathrm{mg} / \mathrm{kg}$ and that using higher doses might cause sustained reduction in cardiac function probably related to the negative inotropic effect [38]. We can only speculate whether reduced end-systolic and diastolic volumes and mixed central venous oxygen saturation in the esmolol group reflects the high esmolol dose used. On the other hand, the trend towards more frequent and prolonged need for dobutamine support in the esmolol group could also be related to a higher total $ß$ adrenergic load resulting in the trend towards higher heart rates in the esmolol group.

Many promising drugs have failed to successfully translate from the laboratory to human trials, and the lack of clinically relevant models is considered to be an important limitation to preclinical research [39]. Clinical cardiac arrest research on the other hand is often limited by the lack of specificity, as peri-arrest factors are often unknown during resuscitation. Clinical studies inherently include patient cohorts consisting of both patients who are easily resuscitated by early defibrillation and patients who are well beyond resuscitation, in addition to the sub-group of patients where an intervention has the potential to improve outcome. Another typical challenge for clinical studies is the heterogeneity of causes of arrest among included patients, resulting in different effects of interventions tested [40]. As an example, interventions might improve haemodynamic and outcome in patients with myocardial infarction, but not in patients with cardiac arrhythmias of non-ischemic origin. Thus, a stronger linkage between specific clinical cardiac arrest phenotypes and tailored preclinical models may improve the relevance of preclinical cardiac arrest studies [41].

\section{Limitations}

There are several limitations worth mentioning. Firstly, as with any animal experimental model, it will never fully replicate the heterogeneous clinical setting. Although we induced acute myocardial infarction with our ischaemic model, animals were young and otherwise healthy prior to our experiments. Similarly, initiation of low-flow VA-ECMO does not represent the physiology during manual CPR because chest compressions provide a different effect on vital perfusion through their effect on intrathoracic pressure, venous pressure and pulsatile flow, thereby affecting the haemodynamic response of esmolol and epinephrine. However pilot testing indicated we would be unable to resuscitate all animals with prolonged arrests using manual or mechanical CPR, and we therefore prioritized a resuscitation strategy that would enable initial resuscitation with significant post-arrest myocardial dysfunction instead of more commonly used clinical strategies. Anatomical differences and variations in balloon placement in the coronary arteries might also confound infarct size and post-arrest cardiac function, although great care was taken to standardize procedures and minimize these effects. Additionally, administration of a vasopressor such as noradrenaline or vasopressin alone has been tested before [42], and could perhaps have added insight as a third group in our study. Finally, although we included ten animals in each group, fewer animals than expected were resuscitated leading to an 
underpowered evaluation of post-arrest cardiac output. However, since there was no clear signal that the "single-dose esmolol" was effective in improving post-arrest myocardial function, we believe it is unlikely that additional experiments would add significant insight. We propose evaluating repeated doses or continuous infusion of esmolol might be more promising avenues to pursue in future experimental exploration of esmolol.

\section{Conclusions}

We observed similar post-arrest cardiac output with and without a single dose of esmolol prior to adrenaline administration during low-flow VA-ECMO in an ischaemic cardiac arrest pig model. While not sufficiently powered to provide a definitive answer, the similarity between the two groups indicates other avenues or models should be encouraged.

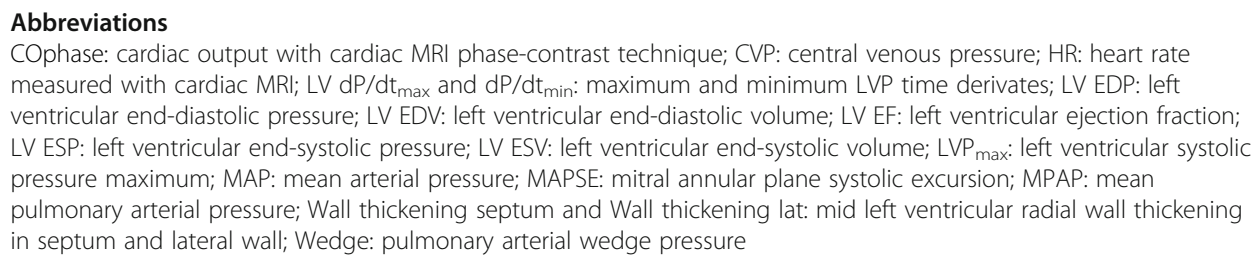

\section{Acknowledgements}

This work was performed at The Intervention Center, Oslo University Hospital. The authors thank the staff at The Intervention Center for assistance during the experiments, Itai Schalit for valuable support in data collection and Christian Eek for assistance during the experiments. We also thank the animal research veterinary and the staff at the Department of Comparative Medicine for care of the animals.

\section{Authors' contributions}

HK, HB, PSH and TMO analysed and interpreted data. HK and TMO drafted the manuscript. All authors have been involved in planning and study design, and all but JFB (deceased) have read and approved the final manuscript.

\section{Funding}

The study was supported by strategic grants awarded Oslo Cardiopulmonary Resuscitation Research (OSCAR) Network by Oslo University Hospital, Department of Research and Development and the Intervention Center, Oslo University Hospital, South-Eastern Norway Regional Health Authority and Laerdal Foundation. The funding bodies had no involvement in the study design, in the collection, analysis or interpretation of data.

\section{Availability of data and materials}

The datasets used and analysed during the current study are available from the corresponding author on reasonable request.

\section{Ethics approval}

The study was approved by the Norwegian National Animal Research Authority (Norwegian Food Safety Authority). The personnel involved in the experiments were certified with Federation of Laboratory Animal Science Associations category C [43], and the study was performed in agreement with the European Convention for the Protection of vertebrate Animals used for Experimental and Other Scientific Purposes (European Council, ETS No. 170) [44] and the ARRIVE guidelines [45].

Consent for publication

Not applicable.

\section{Competing interests}

The authors declare that they have no competing interests.

\section{Author details}

'Department of Research and Development, Oslo University Hospital, PB 4956 Nydalen, N-0424 Oslo, Norway. ${ }^{2}$ The Intervention Center, Department of Anaesthesiology, Oslo University Hospital, Oslo, Norway. ${ }^{3}$ Department of Anaesthesiology, Oslo University Hospital, Oslo, Norway. ${ }^{4}$ Institute of Clinical Medicine, University of Oslo, Oslo, Norway. ${ }^{5}$ Department of Cardiology, Oslo University Hospital, Oslo, Norway. ${ }^{6}$ Oslo Cardiopulmonary Resuscitation Research Network, Oslo, Norway. 
Received: 31 August 2019 Accepted: 31 October 2019

\section{Published online: 04 December 2019}

\section{References}

1. Grasner JT et al (2016) EuReCa ONE-27 Nations, ONE Europe, ONE Registry: A prospective one month analysis of out-ofhospital cardiac arrest outcomes in 27 countries in Europe. Resuscitation 105:188-195

2. Perkins GD et al (2015) European Resuscitation Council Guidelines for Resuscitation 2015: Section 2. Adult basic life support and automated external defibrillation. Resuscitation 95:81-99

3. Soar J et al (2015) European Resuscitation Council Guidelines for Resuscitation 2015: Section 3. Adult advanced life support. Resuscitation 95:100-147

4. Pearson JW, Redding JS (1963) EPINEPHRINE IN CARDIAC RESUSCITATION. Am Heart J 66:210-214

5. Michael JR et al (1984) Mechanisms by which epinephrine augments cerebral and myocardial perfusion during cardiopulmonary resuscitation in dogs. Circulation 69:822-835

6. Otto CW, Yakaitis RW (1984) The role of epinephrine in CPR: a reappraisal. Ann Emerg Med 13:840-843

7. Perkins GD et al (2018) A Randomized Trial of Epinephrine in Out-of-Hospital Cardiac Arrest. N Eng J Med 379:711-721

8. Jacobs IG et al (2011) Effect of adrenaline on survival in out-of-hospital cardiac arrest: A randomised double-blind placebo-controlled trial. Resuscitation 82:1138-1143

9. Olasveengen TM et al (2009) Intravenous drug administration during out-of-hospital cardiac arrest: A randomized trial. JAMA 302:2222-2229

10. Ditchey RV, Lindenfeld J (1988) Failure of epinephrine to improve the balance between myocardial oxygen supply and demand during closed-chest resuscitation in dogs. Circulation 78:382-389

11. Niemann JT et al (1986) Postcountershock pulseless rhythms: response to CPR, artificial cardiac pacing, and adrenergic agonists. Ann Emerg Med 15:112-120

12. Neset $A$ et al (2013) Effects of adrenaline on rhythm transitions in out-of-hospital cardiac arrest. Acta Anaesthesiol Scand 57:1260-1267

13. Angelos MG et al (2008) Cardiovascular response to epinephrine varies with increasing duration of cardiac arrest. Resuscitation 77:101-110

14. Tang $W$ et al (1995) Epinephrine increases the severity of postresuscitation myocardial dysfunction. Circulation 92 : 3089-3093

15. Jingjun $L$ et al (2009) Effect and mechanism of esmolol given during cardiopulmonary resuscitation in a porcine ventricular fibrillation model. Resuscitation 80:1052-1059

16. Zhang Q, Li C (2013) Combination of epinephrine with esmolol attenuates post-resuscitation myocardial dysfunction in a porcine model of cardiac arrest. PloS One 8:e82677

17. Bassiakou E et al (2008) Atenolol in combination with epinephrine improves the initial outcome of cardiopulmonary resuscitation in a swine model of ventricular fibrillation. Am J Emerg Med 26:578-584

18. Cammarata G et al (2004) Beta1-adrenergic blockade during cardiopulmonary resuscitation improves survival. Crit Care Med 32:S440-S443

19. Ditchey RV, Rubio-Perez A, Slinker BK (1994) Beta-adrenergic blockade reduces myocardial injury during experimental cardiopulmonary resuscitation. J Am Coll Cardiol 24:804-812

20. Killingsworth CR et al (2004) Short-acting beta-adrenergic antagonist esmolol given at reperfusion improves survival after prolonged ventricular fibrillation. Circulation 109:2469-2474

21. Huang $L$ et al (2005) Carvedilol mitigates adverse effects of epinephrine during cardiopulmonary resuscitation. J Cardiovasc Pharmacol Ther 10:113-120

22. Angaran DM, Schultz NJ, Tschida VH (1986) Esmolol hydrochloride: an ultrashort-acting, beta-adrenergic blocking agent. Clinical pharmacy 5:288-303

23. Driver BE et al (2014) Use of esmolol after failure of standard cardiopulmonary resuscitation to treat patients with refractory ventricular fibrillation. Resuscitation 85:1337-1341

24. Bergan HA et al (2015) Successful ECMO-cardiopulmonary resuscitation with the associated post-arrest cardiac dysfunction as demonstrated by MRI. Intensive Care Med Exp 3:61

25. Schulz-Menger J et al (2013) Standardized image interpretation and post processing in cardiovascular magnetic resonance: Society for Cardiovascular Magnetic Resonance (SCMR) board of trustees task force on standardized post processing. J Cardiovasc Magn Reson 15:35

26. Kilner PJ, Gatehouse PD, Firmin DN (2007) Flow measurement by magnetic resonance: a unique asset worth optimising. J Cardiovasc Magn Reson 9:723-728

27. Heiberg E et al (2010) Design and validation of Segment--freely available software for cardiovascular image analysis. BMC Med Imaging 10:1

28. Pischke SE et al (2017) Complement factor 5 blockade reduces porcine myocardial infarction size and improves immediate cardiac function. Basic Res Cardiol 112:20

29. Heusch G, Skyschally A, Schulz R (2011) The in-situ pig heart with regional ischemia/reperfusion - ready for translation. J Mol Cell Cardiol 50:951-963

30. Exner DV et al (1999) Beta-blocker use and survival in patients with ventricular fibrillation or symptomatic ventricular tachycardia: the Antiarrhythmics Versus Implantable Defibrillators (AVID) trial. J Am Coll Cardiol 34:325-333

31. Connolly SJ et al (2006) Comparison of beta-blockers, amiodarone plus beta-blockers, or sotalol for prevention of shocks from implantable cardioverter defibrillators: the OPTIC Study: a randomized trial. JAMA 295:165-171

32. Lee YH et al (2016) Refractory ventricular fibrillation treated with esmolol. Resuscitation 107:150-155

33. Theochari E et al (2008) Selective beta blockade improves the outcome of cardiopulmonary resuscitation in a swine model of cardiac arrest. Ann Ital Chir 79:409-414

34. Li Z et al (2019) Selective beta-blocker esmolol improves cerebral cortex microcirculation in a swine ventricular fibrillation model. J Cell Biochem 120:3679-3688

35. Strohmenger HU et al (1999) Effects of the specific bradycardic agent zatebradine on hemodynamic variables and myocardial blood flow during the early postresuscitation phase in pigs. Resuscitation 42:211-220 
36. Zoerner $\mathrm{F}$ et al (2015) Milrinone and esmolol decrease cardiac damage after resuscitation from prolonged cardiac arrest. Acta Anaesthesiol Scand 59:465-474

37. Lu J et al (2015) Combining Epinephrine and Esmolol Attenuates Excessive Autophagy and Mitophagy in Rat Cardiomyocytes After Cardiac Arrest. J Cardiovasc Pharmacol 66:449-456

38. Pirk J et al (1999) The effect of the ultrashort beta-blocker esmolol on cardiac function recovery: an experimental study. Eur J Cardiothorac Surg 15:199-203

39. Cherry $\mathrm{BH}$ et al (2015) Modeling cardiac arrest and resuscitation in the domestic pig. World journal of critical care medicine 4:1-12

40. Finn J et al (2019) Adrenaline and vasopressin for cardiac arrest. The Cochrane database of systematic reviews 1: cd003179

41. Uray T et al (2018) Phenotyping Cardiac Arrest: Bench and Bedside Characterization of Brain and Heart Injury Based on Etiology. Crit Care Med 46:e508-e515

42. Prengel AW et al (2005) Effects of combined administration of vasopressin, epinephrine, and norepinephrine during cardiopulmonary resuscitation in pigs. Crit Care Med 33:2587-2591

43. Guillen J (2012) FELASA guidelines and recommendations. J Am Assoc Lab Anim Sci 51:311-321

44. (https://www.coe.int/en/web/conventions/full-list/-/conventions/treaty/123) ECftPovAufEaOSPaja European Convention for the Protection of vertebrate Animals used for Experimental and Other Scinetific Purposes. In: Editor (ed)^(eds) Book European Convention for the Protection of vertebrate Animals used for Experimental and Other Scinetific Purposes. City, pp. Accessed 11 Apr 2019

45. Kilkenny $C$ et al (2010) Improving bioscience research reporting: the ARRIVE guidelines for reporting animal research. PLOS Biol 8:e1000412

\section{Publisher's Note}

Springer Nature remains neutral with regard to jurisdictional claims in published maps and institutional affiliations.

\section{Submit your manuscript to a SpringerOpen ${ }^{\circ}$} journal and benefit from:

- Convenient online submission

- Rigorous peer review

- Open access: articles freely available online

- High visibility within the field

Retaining the copyright to your article

Submit your next manuscript at $\boldsymbol{\nabla}$ springeropen.com 